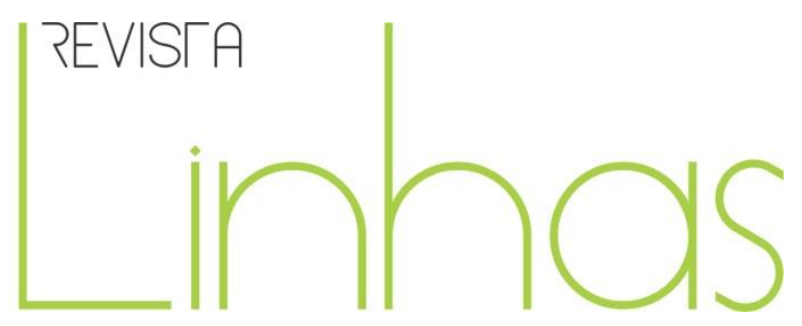

\title{
Relazione educativa, valutazione e lavoro di equipe
}

\begin{abstract}
Riassunto
Prendere in esame le dimensioni organizzative e relazionali di un contesto educativo significa analizzare quei fattori che, pur disegnandone la fisionomia educativa, molto spesso rimangono impliciti e irriflessi. In tal senso, per acquisire e rigenerare nuove consapevolezze circa le implicazioni pedagogiche delle pratiche quotidiane, diviene sempre più necessario promuovere e sostenere $i$ processi di riflessione all'interno di ciascuna èquipe pedagogica, mediante una pluralità di prospettive e metodologie. In quest'ottica, la valutazione formativa del contesto educativo si configura come un processo dialogico e transattivo, nel quale ciascun gruppo di lavoro è attivo nell'interrogare i processi realizzati e attivati con i bambini, al fine di condividere e negoziare i significati, le finalità e i valori che li orientano e, quindi, acquisire maggiori consapevolezze, superando così un'accezione meramente certificativa e classificatoria dell'atto valutativo. In particolare, il poter osservare, descrivere e valutare la qualità della relazione educativa, a partire dall'analisi di alcuni frammenti video riguardanti gli scambi comunicativi realizzati con i bambini, diviene per ciascuna èquipe educativa un'occasione formativa particolarmente rilevante, in quanto consente di soffermarsi su aspetti cardine dell'agire educativo, ma che rischiano di restare inglobati nel flusso delle routine quotidiane e relegati alla discrezionalità del singolo insegnante. Scelte organizzative e modalità relazionali che possono essere ulteriormente interrogate $\mathrm{e}$ problematizzate intrecciando tali metodologie con approcci fondati sull'impiego di strategie di scrittura riflessiva, realizzando così con ogni gruppo educativo dei percorsi formativi, nei quali sguardi e saperi differenti si confrontano per co-costruire repertori di significati condivisi e generare nuove consapevolezze.
\end{abstract}

Parole chiave: Relazione Educativa. Valutazione Formativa. Riflessione. Collegialità. Educazione Prescolare.

\section{Antonio Gariboldi}

Università degli Stud di Modena e

Reggio Emilia - Italia

antonio.gariboldi@unimore.it

\section{Antonella Pugnaghi}

Università degli Stud di Modena e

Reggio Emilia - Italia

antonella.pugnaghi@unimore.it

\section{Para citar este artigo: \\ GARIBOLDI, Antonio; PUGNAGHI, Antonella. Relazione educativa, valutazione e lavoro di equipe. Revista}

Linhas. Florianópolis, v. 19, n. 40, p. 116-137, maio/ago. 2018. 


\section{Teacher-child educational collegiality}

\section{Abstract}

Taking into consideration the organizational and relational dimensions of an educational context means analyzing those factors that, although they draw its educational physiognomy, very often remain implicit and unaware. In this sense, to acquire and regenerate new awareness about the pedagogical implications of everyday practices, it becomes increasingly necessary to promote and support the processes of reflection within each pedagogical equipe, through a plurality of perspectives and methodologies. In this perspective, the educational evaluation of the context is outlined as a dialogic and transactive process, in which each working group is active in interrogating the processes implemented and activated with the children, in order to share and negotiate the meanings, the purposes and values that guide them and, therefore, to acquire greater awareness, thus overcoming a merely certification and classificatory view of the evaluation. In particular, being able to observe, describe and evaluate the quality of the teacher-child relationship, starting from the analysis of some video concerning the dialogues realized with the children, becomes an important training opportunity for each educational equipe to dwell on key aspects of educational action, but that they risk becoming embedded in the flow of daily routines and bound to the discretion of the individual teacher. Organizational choices and relational modalities that can be further interrogated and problematized by intertwining these methodologies with approaches based on the use of reflective writing strategies, thus realizing with each educational group the training paths, in which different perspectives and knowledge are compared to co-construct repertoires of shared meanings and generate new awareness.

Keywords: Teacher-Child Relationship. Educational Evaluation. Professional Reflectivity. Collegiality. Early Childhood Education.

\section{Relação educativa, avaliação e trabalho de equipe}

\section{Resumo}

Levar em consideração as dimensões organizacionais e relacionais de um contexto educacional significa analisar aqueles fatores que, ao mesmo tempo em que desenvolvem sua fisionomia educacional, muitas vezes permanecem implícitos e irreflexivos. Nesse sentido, para adquirir e regenerar novas conscientizações sobre as implicações pedagógicas das práticas cotidianas, torna-se cada vez mais necessário promover e apoiar os processos de reflexão dentro de cada equipe pedagógica, através de uma pluralidade de perspectivas e metodologias. Deste ponto de vista, a avaliação formativa do contexto educacional é configurada como um processo dialógico e transacional, em que cada grupo de trabalho é ativo em interrogar os processos realizados e ativados com as crianças, a fim de partilhar e negociar os significados, propósitos e valores que os orientam e, portanto, adquirir uma maior conscientização, superando assim apenas um aspecto de certificação e classificação da avaliação. Em particular, ser capaz de observar, descrever e avaliar a qualidade da relação educativa, a partir da análise de alguns fragmentos de vídeo em relação aos intercâmbios de comunicação realizados com as crianças, tornase para cada equipe educacional uma oportunidade de formação particularmente importante, pois permite analisar aspectos-chave da ação educacional, mas que podem permanecer incorporados no fluxo das rotinas diárias e relegados ao critério de cada professor. Escolhas organizacionais e modalidades relacionais que podem ser ainda interrogadas e problematizadas entrelaçando estas metodologias com abordagens baseadas no uso de estratégias de escrita reflexiva, criando assim com cada grupo educacional percursos de formação, em que diferentes olhares e conhecimentos se confrontam para co-construir repertórios de significados compartilhados e gerar novas consciências.

Palavras-chave: Relação Educativa. Avaliação Formativa. Reflexão. Colegialidade. Educação PréEscolar. 


\section{Curricolo e pedagogia latente nella scuola dell'infanzia: una prospettiva riflessiva}

L'attenzione progettuale all'organizzazione del contesto educativo - e quindi a forme di educazione che mettono al centro il protagonismo del bambino - si è gradualmente sviluppata nell'ambito di una impostazione pedagogica che ha concepito il curricolo formativo della scuola dell'infanzia come orientato in senso ecologico. L'affermarsi nel campo dell'educazione prescolare delle teorie sulla costruzione sociale dell'intelligenza e di una interpretazione ecologica dello sviluppo infantile, che lo intende come dinamicamente legato alle caratteristiche del sistema d'interazione sociale ed alle proprietà dell'ambiente di cui il soggetto fa parte, pone come presupposto dell'azione educativa la dimensione sistemica del processo evolutivo e il ruolo attivo e connettivo svolto dal bambino all'interno del contesto a cui partecipa.

Una tale concezione implica, sul piano della formazione, una maggiore considerazione delle componenti ambientali e sociali, la valorizzazione delle modalità di autorganizzazione cognitiva e socio-affettiva del bambino ed una più attenta riflessione sui possibili legami tra gli elementi formali e informali del processo educativo. In questo senso, nell'ambito di un approccio pedagogico che valorizza in chiave formativa il gioco, la vita di relazione e i processi di esplorazione e scoperta e che attribuisce in primo luogo all'insegnante un ruolo di regia ambientale, appare necessario sviluppare consapevolezza su come l'intreccio delle variabili organizzative e relazionali - l'organizzazione dello spazio, del tempo e dei gruppi e le dinamiche relazionali tra adulto e bambini e tra bambini contribuisca a determinare il significato e la qualità delle esperienze educative. II rischio è, infatti, che le componenti di contesto vengano intese come una sorta di sfondo della pratica didattica, senza considerare in modo approfondito il ruolo fondamentale che assumono nel creare la fisionomia dei processi formativi.

La riflessione sulla dimensione organizzativa e relazionale del setting educativo, sulla tendenza che hanno tali dimensioni a connotarsi come elementi di pedagogia irriflessa e quindi a orientare in maniera implicita e latente il funzionamento del curricolo,

comporta la necessità di rendere oggetto di analisi e valutazione da parte dell'équipe educativa quelle variabili di contesto che, in modo più o meno consapevole, visibile e 
progettato, concorrono a definire una specifica realtà scolastica. Occorre quindi fare riferimento a tutti quei fattori materiali, relazionali e simbolici che determinano un contesto educativo, molti dei quali sfuggono ad una deliberazione cosciente, eccedono la pratica programmatoria e tendono a delineare un più esteso e diffuso curricolo invisibile 0 nascosto.

L'attenzione a quelle dimensioni nascoste, anche di tipo relazionale e simbolico, che disegnano la fisionomia di un contesto educativo e che hanno una notevole ricaduta formativa sull'apprendimento di habitus mentali, modelli di comportamento e competenze implica il richiamo alla nozione di pedagogia latente (Becchi, 1978). Nozione finalizzata a circoscrivere ed a mettere in risalto quegli elementi impliciti e inespressi del setting educativo che sfuggono alla pianificazione dell'intervento formativo, ma che hanno un'influenza significativa nel modellare gli atteggiamenti e le condotte infantili e nello stimolare lo sviluppo delle differenti capacità.

E' dunque presente, in diversa misura nelle varie situazioni educative, una pedagogia latente connotata da criteri diffusi e modalità di trasmissione implicite, legati soprattutto ad aspetti organizzativi e relazionali, che ha una significativa ricaduta formativa a lungo termine sulla creazione di disposizioni affettive, abiti mentali e comportamentali. Tale presenza diffusa nelle scelte organizzative e nell'ordinaria azione educativa "presuppone la necessità di considerare quanto nel corso della quotidianità rimane "latente": ciò che si nasconde nell'ovvietà, ciò a cui non si pensa perché abituale, ciò che si tende a dare per scontato" (Palmieri, 2014, p. 16). Generare o rigenerare consapevolezze sugli assetti organizzativi e sulle condotte più ovvie rappresenta quindi un'esigenza mai definitivamente soddisfatta del lavoro di un'equipe educativa e richiede, di conseguenza, di far emergere e condividere il significato di azioni che si riproducono in modo quasi automatico, implica un lavoro costante, il ritornare ricorsivamente e riflessivamente sugli atti e contesti che connotano l'agire quotidiano. Presuppone il creare le condizioni (tempi, occasioni e strumenti) per fermarsi e per recuperare la dimensione della presenza sui modi con cui ogni giorno si opera. Perciò si può dire che "guadagnare una professionalità evoluta significa fondarla su risorse di apprendimento che consentano di transitare da zone di preriflessività a zone più consapevoli. Il guadagno 
di un pensiero critico-riflessivo è la condizione per lo sviluppo professionale" (Fabbri, 2007, p. 79).

Quanto messo in rilievo sollecita, dunque, ad assumere una prospettiva riflessiva sul curricolo della scuola dell'infanzia, "perché la costruzione del curricolo impone ai docenti una continua valutazione della coerenza tra scelte didattiche, principi pedagogici ed esiti raggiunti nei tempi brevi e in quelli lunghi, alla ricerca di un giusto equilibrio tra razionalità metodologica e tensione etica, ma soprattutto per limitare il peso di aspetti impliciti e irriflessi che possono condizionare negativamente il funzionamento del curricolo" (Franceschini, Borin, 2014, p. 111).

Sotto questo profilo la valutazione del contesto educativo si configura come lo strumento di un approccio situato e riflessivo alla formazione della équipe educativa, in quanto mette al centro dell'analisi e della riflessione la coerenza tra il dichiarato progettuale e l'agito educativo, consentendo di mettere in luce le implicazioni pedagogiche delle pratiche quotidiane e quindi stimolando la negoziazione di significato nell'ambito del gruppo, l'apprendimento trasformativo e la costruzione di una progettualità educativa più esplicita e condivisa.

Si parla, in questo caso, di un processo di valutazione formativa fortemente caratterizzato in senso dialogico e transattivo, che si propone di cogliere i diversi aspetti di una realtà dinamica nella molteplicità dei punti di vista, evidenziando i rapporti d'interdipendenza tra i vari elementi del contesto per poter ragionare sulla complessità di una situazione educativa. Si tratta, di conseguenza, di una forma di indagine autoriflessiva fondata sul coinvolgimento e la partecipazione degli insegnanti, che implica procedure sistematiche di autoanalisi della realtà educativa e che è orientata alla condivisione di un repertorio interpretativo e valutativo.

Se la costruzione di consapevolezze e l'evoluzione di una realtà educativa sono legate ad un processo di apprendimento che avviene all'interno di una cornice partecipativa, cioè "nel contesto di specifiche forme di copartecipazione sociale" (Hanks, 2006, p. 10) che implicano la possibilità di negoziare significati e prendere decisioni, allora la valutazione può assumere un carattere realmente formativo solo se attiva in profondità 
il gruppo di lavoro coinvolgendolo in una pratica sociale di ricerca. In questo senso la qualità dei processi educativi non può fondarsi su una logica ispettiva di controllo, ma deve reggersi su una logica di assunzione di responsabilità che implica partecipazione e sviluppo della capacità di autoregolazione. Imparare a valutare e valutarsi significa imparare ad attivare processi di autoanalisi e quindi apprendere modalità di controllo della propria azione per evitare che si caratterizzi nei termini di un'operatività irriflessa.

In questo senso un'area di sviluppo professionale che richiede continuamente esame e pensiero, e quindi una presenza riflessiva nelle situazioni che si connotano come consuetudinarie, è la relazione educativa. Una dimensione essenziale del "modo di fare scuola" (Cappa, 2014) che evoca e rimanda alla complessità della nozione di intenzionalità educativa, intesa come concetto non riducibile unicamente agli obiettivi razionalmente definiti e perseguiti, ma come "sistemi di ragioni" che in modo parzialmente inconsapevole e intuitivo fondano l'agire educativo del soggetto e sono connessi all'insieme della sua esperienza e alla "storia" che più in generale lo riguarda (Colicchi, 2005). Così appare fondamentale dotarsi di momenti e strumenti che assumano come oggetto di analisi le forme di regolazione del campo intersoggettivo insegnantebambino/i, per sollecitare la disamina di ciò che a volte sembra "più conosciuto che pensato", stimolando un processo di messa in discussione delle motivazioni del proprio fare, di esplicitazione e condivisione dei significati educativi impliciti e dunque di incremento collettivo dei livelli di consapevolezza pedagogica.

Lavorare in chiave formativa sulla relazione educativa implica allora valutarla, nel senso di comprenderla e attribuirvi senso e valore pedagogico, considerandola come elemento strutturale del contesto educativo, cioè analizzandola nelle sue interdipendenze funzionali con l’organizzazione di spazi, materiali, tempi e regole delle situazioni/proposte educative. Qui la valutazione, che anche attraverso l'attivazione di dinamiche di contrasto/conflitto si pone l'obiettivo della revisione delle "forme ovvie" di relazione, è legata alla creazione di un contesto di scambio e discussione che, a partire dalla rilevazione e analisi di elementi operativi, consente il confronto tra una pluralità di prospettive, facendo emergere e rendendo oggetto di critica le diverse rappresentazioni di "buona relazione educativa". 


\section{Relazione educativa: è possibile valutarne la qualità?}

Cercare di comprendere la complessità che contraddistingue ciascun contesto educativo significa prendere in esame una molteplicità di aspetti (materiali, organizzativi, didattici, simbolici e valoriali) tra loro strettamente interconnessi, ma vuol dire soprattutto interrogarsi circa la qualità delle dinamiche relazionali esperite quotidianamente da ciascun bambino all'interno di tale realtà.

La relazione educativa, intesa come una storia di interazioni (Hinde, 1981), ossia una storia di microscambi che avviene in contesti particolari, adottando stili particolari (Mantovani, 1989), si configura come una relazione non banale, intrinsecamente complessa, principale indicatore del buon funzionamento del contesto stesso.

In particolare, per esplorare in maniera rigorosa la natura multidimensionale di tale dimensione occorre adottare una prospettiva eco-sistemica (Pianta, 1999) nella quale la relazione educativa si viene a delineare come un sistema aperto, complesso, attraversato da specifiche dinamiche, più o meno evolute, più o meno consapevoli, origine di quell'infrastruttura evolutiva su cui si costruiscono le esperienze scolastiche.

Infatti, pur riconoscendo in tale relazione una dimensione essenziale, nevralgica dell'esperienza educativa di ciascun contesto prescolare, troppo spesso essa rischia di restare imprigionata nella discrezionalità dei pensieri e delle azioni del singolo educatore, senza divenire oggetto di discussione e formazione all'interno del gruppo di lavoro educativo.

Pertanto, poter osservare, descrivere e valutare in maniera rigorosa la qualità delle dinamiche relazionali implementate quotidianamente all'interno dei diversi contesti educativi diviene un'occasione per i vari soggetti coinvolti per discutere di un aspetto nevralgico dell'esperienza infantile nella sua sostanza, senza rischiare di scadere in interpretazioni impressionistiche e banalizzanti.

In tal senso, la valutazione della qualità della relazione educativa ha costituito l'intento principale di numerosi percorsi di ricerca internazionali (Pianta et al., 2005, 2009; LoCasale-Crounch et al., 2007; Curby et al., 2009; Downer et al., 2012; Cabell et al., 2013; 
Hamre et al., 2013; Pattison et al., 2014) e recentemente anche di indagini condotte in ambito europeo (Cadima et al., 2010; Pakarinen et al., 2010, 2012; Salminen et al., 2012; von Suchodoletz et al., 2014), ricerche nelle quali si è adottato uno specifico dispositivo di osservazione e valutazione: Classroom Assessment scoring system (Pianta, La Paro, Hamre, 2008).

Nello specifico, allontanandosi da ogni tipologia di osservazione target child, tale scala di valutazione indaga la relazione educativa focalizzando l'attenzione su dei costrutti chiave generali, operazionalizzati in descrizioni di pattern comportamentali olistici, al fine di rilevare l'ecologia relazionale complessiva caratterizzante ciascun contesto educativo prescolare (Dower, Sabor, Hamre, 2010).

Figura 1

I domini e le dimensioni del CLASS Infant, Toddler e Pre-k (Fonte: Vitiello, Hadden, 2014, p.10)

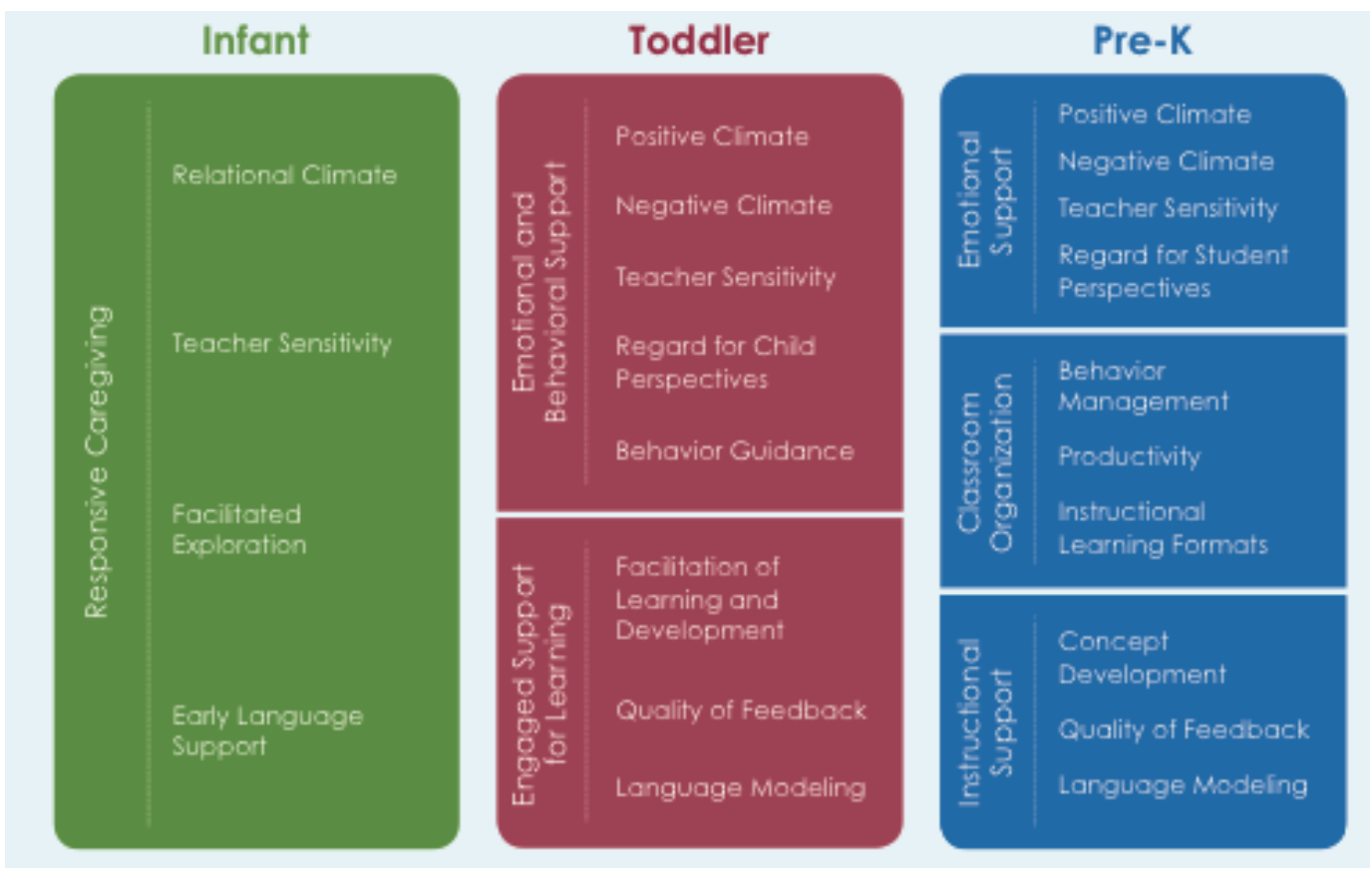

Senza voler entrare nello specifico, come si evince dalla figura n.1, la strutturazione interna del dispositivo si articola in diversi domini a seconda del contesto educativo di volta in volta indagato, consentendo di sondare la relazione educativa secondo una logica 
di continuità $0 / 6$, indicando alcuni costrutti chiave trasversali che, con connotazioni differenti in base all'età dei bambini, presentano però radici e significati condivisi.

In tal senso, occorre precisare che, affinché si realizzino dei reali percorsi di valutazione formativa, occorre non limitarsi ad un utilizzo tecnicistico dello strumento di valutazione adottato, correndo così il rischio di scadere in una fruizione acritica, ma diviene necessario soffermarsi a problematizzare la specifica prospettiva teorico-culturale in esso espressa.

In questa prospettiva, mettere in discussione e riflettere sui criteri di valutazione delineati in questo strumento significa incentivare ciascun partecipante ad interrogarsi sulle convinzioni, rappresentazioni e premesse epistemiche che guidano spesso inconsapevolmente la propria esperienza professionale. Infatti, se da un lato, condividendo dei descrittori e dei criteri di qualità comuni, fondati teoricamente e validati empiricamente in numerosi percorsi di ricerca, tale strumento di valutazione viene introdotto nel gruppo di lavoro come un sapere di orizzonte dal valore provvisorio (Mortari, 2009), un filtro utile per scandagliare anche gli aspetti più emotivi e irrazionali che contraddistinguono le dinamiche relazioni, dall'altro occorre sempre problematizzare la specifica prospettiva teorica su cui esso si fonda, riflettendo e mettendo in discussione il modello di relazione educativa in esso delineato. Si tratta, dunque, di predisporre, all'interno dei percorsi di valutazione formativa, dei momenti nei quali diviene possibile alimentare un dialogo fecondo tra il sapere formale espresso nella scala di valutazione e proposto dal ricercatore e il sapere esperienziale elaborato dai diversi professionisti coinvolti, "in quanto condizione essenziale per rendere il sapere formale vividamente utile sul campo e il sapere della pratica criticamente fondato" (Mortari, 2009, p.107).

In questa prospettiva, a partire da un percorso di ricerca di ricerca intitolato "Pattern educativi nella scuola dell'infanzia. Una ricerca nelle scuole dell'infanzia della provincia di Modena", negli ultimi anni si sono ideati e implementati diversi percorsi formativi rivolti, da un lato, agli studenti del Corso di Laurea in Scienze della Formazione primaria presso l'Università degli Studi di Modena e Reggio Emilia, mediante la strutturazione di alcuni laboratori e, dall'altro lato, a diversi gruppi di lavoro educativi 
(insegnanti-educatori, coordinatori e dirigenti), tramite la realizzazione di percorsi di valutazione formativa.

Nello specifico, in queste ultime indagini valutative, l'impiego del CLASS da parte dei diversi professionisti coinvolti viene a caratterizzarsi quale specchio professionale, grazie al quale diviene possibile esplicitare i significati profondi, sottesi alle diverse modalità relazionali osservate, ma anche confrontarsi e problematizzare la filosofia di qualità, il modello relazionale descritto ed esplicitato in tale scala di valutazione.

Per questo motivo, diviene interessante prendere in esame alcuni spunti di riflessione emersi nei diversi incontri di restituzione realizzati con i vari professionisti coinvolti, contesti formativi questi ultimi nei quali, sottoponendo al vaglio critico ciò che accade quotidianamente nei servizi, diviene possibile esplicitare, condividere e problematizzare quegli alfabeti, repertori conoscitivi di riferimento e assunzioni tacite che ciascuna èquipe educativa elabora nel corso del tempo, al fine di acquisirne una maggiore consapevolezza e guardare all'esperienza con uno sguardo nuovo (Mortari, 2003).

\subsection{Indagare la cura educativa con il CLASS}

Pur riconoscendo le specificità che contraddistinguono i diversi servizi per l'infanzia, una dimensione essenziale che accomuna e contraddistingue le dinamiche relazionali che si realizzano in tali contesti riguarda il costrutto chiave di cura educativa.

Se guardiamo alla cura come alla matrice di uno stile relazionale che tiene insieme corpo, mente ed emozioni (Terzi, 2017), occorre allora non solo prestare attenzione alle tonalità e ai vissuti emotivi che caratterizzano una buona relazione educativa, ma cogliere anche quanto e in che modo tali relazioni siano importanti per l'apprendimento.

In questo senso, nell'articolazione interna del dispositivo di valutazione adottato tale costrutto si ritrova principalmente nel dominio riconducibile al Supporto Emotivo, dimensione quest'ultima che, facendo esplicito riferimento alla teoria dell'attaccamento e dell'autodeterminazione (Bowlby, 1969; Connell, Wellborn, 1991), riguarda il grado in cui i bambini fanno esperienza di contesti educativi contrassegnati da un clima relazionale positivo, in cui si incentivano i rapporti tra pari e dove le insegnanti/educatrici prestano 
attenzione agli interessi, bisogni individuali di ciascun bambino, fornendo loro delle reali opportunità per conversare e lavorare autonomamente. Tali condizioni relazionali contribuiscono a creare quel senso di sicurezza che incentiva in ciascun bambino il desiderio della scoperta, andando ad alimentare la sua inesauribile sete di conoscenza (Dower, Sabol, Hamre, 2010).

A tal proposito, essere in linea con quanto dichiarato nello strumento di valutazione significa per l'adulto adottare uno stile educativo fondato sull'ascolto, sull'intervento indiretto, un regista attento a curare le condizioni organizzative e relazionali affinché tutti i bambini possano partecipare attivamente alle proprie esperienze di apprendimento, promuovendo dei reali processi di costruzione attiva dei saperi.

In particolare, analizzando i criteri di qualità riconducibili a tale ambito di indagine, esplicitati nella versione del CLASS riguardante i contesti prescolari frequentati da bambini di età compresa tra i 3 e i 5 anni (pre-kindergarden), si richiama l'attenzione sulla centralità del bambino e sull'apertura e l'ascolto, quali modalità relazionali che devono contraddistinguere l'atteggiamento quotidiano di ciascun professionista coinvolto. "Penso che per svolgere bene il proprio ruolo ciascun insegnante deve rimanere disponibile e aperto a ciò che accade ogni giorno. Perché se dai per scontato che tutto andrà secondo i tuoi piani perdi la parte migliore del nostro lavoro" (Insegnante).

Per gli insegnanti coinvolti aver cura degli interessi mostrati dai bambini e portare rispetto ai loro pensieri significa anche mettere in campo una disponibilità autentica, consapevole del proprio ruolo, in grado di rilevare i continui spunti offerti dai bambini e di ricondurli in una narrazione comune e coerente. "Se tu non vuoi appiattire tutto a percorsi lineari, preconfezionati che trovi in tutti i manuali... Non devi fare dei pensieri strani o ricercare chissà quale innovazione, ma dare valore a quello che accade nella quotidianità, ricercare insieme ai bambini senza dar nulla per scontato. Si tratta di vivere tutto con il loro sguardo curioso, pieno di perché... dove nulla è lineare. Non devo aver paura dell'imprevisto, anzi è proprio quello che mi può salvare dal fare ogni giorno le stesse cose" (Insegnante). 
In questa prospettiva, il prestare attenzione e ascolto ai bisogni e agli interessi dei bambini si traduce in un'apertura intenzionale che non sfocia in alcuna forma di spontaneismo, in quanto fondata su un pensiero progettuale in grado di sostenere nei bambini la loro autonoma lettura del mondo. In tal senso, come sostiene Sergio Neri: "su tutto prevale una formidabile attenzione degli insegnanti ad "ascoltare" i bambini, a leggerne i tentativi, i desideri, le direzioni di crescita, senza perciò forzarli, ma senza nemmeno indulgere nell'attesa del tempo di maturazione" (Neri, 2001, p.133).

In chiave formativa, il poter descrivere e condividere le emozioni e i vissuti che attraversano questi difficili equilibri relazionali all'interno del proprio gruppo di lavoro significa, dunque, riconoscere nella ricerca valutativa non un momento meramente certificativo e classificatorio, ma un'occasione per imparare a interrogare i processi realizzati e attivati con i bambini, per riflettere, condividere e negoziare i significati, le finalità e i valori in modo critico e, quindi, acquisire maggiori consapevolezze.

Tutto ciò assume ancora più importanza se si guarda a queste possibilità formative in una logica di continuità $0 / 6$, predisponendo dunque contesti nei quali supportare e alimentare il confronto tra le diverse professionalità coinvolte (educatori, insegnanti, coordinatori pedagogici e dirigenti scolastici), costringendole a soffermarsi su aspetti che spesso possono essere inglobati nel flusso delle routine quotidiane, incentivandone dunque nuove consapevolezze e continue riflessioni (Savio, 2011; Balduzzi, Manini, 2013).

\subsection{L'utilizzo dei video come dispositivi euristici e formativi}

Per non scadere in vuote retoriche $e$ in sterili ripiegamenti intimistici e autoreferenziali, occorre ancorare la capacità osservativa e interpretativa dei professionisti coinvolti all'analisi puntuale delle diverse esperienze quotidiane, realizzate nei diversi contesti prescolari esaminati.

Per questo motivo, in ogni incontro di restituzione realizzato, oltre a supportare le riflessioni delle figure educative coinvolte con l'utilizzo dello strumento di valutazione (CLASS), usato quale termine di paragone, si è deciso di restituire la complessità delle 
dinamiche relazionali analizzate mediante la visione e l'analisi di alcuni filmati video riguardanti la quotidianità del servizio di volta in volta indagato.

Lontano da ogni forma di riduzionismo tecnicistico e aderendo alla riflessione sui video-casi prospettata da Tochon (2009), l'impiego del video viene a costituirsi quale potente dispositivo euristico, in grado di stimolare la riflessione costruttiva sulla pratica e supportare importanti percorsi di empowerment professionale mediante lo studio e l'analisi dei processi che contraddistinguono ogni evento educativo. A partire dalla visione di alcuni frammenti video diviene, infatti, possibile ri-accostarsi all'esperienza, soffermarsi su diversi aspetti riguardanti non solo gli elementi esteriori quali i comportamenti attuati dai soggetti coinvolti, ma anche aspetti interiori, ossia pensieri e rappresentazioni ad essi connessi (Cescato, 2015).

Riconoscendo, dunque, nel video una forma di narrazione aperta e metacognitiva (Corazza, 2012), si vuole predisporre dei contesti problematizzanti nei quali ciascun partecipante è invitato a scandagliare la complessità relazionale che contraddistingue ciascun evento educativo, al fine di far emergere e condividere i presupposti educativi e gli impliciti teorici che attraversano e sedimentano nelle pratiche quotidiane. In tal senso, poter osservare, descrivere e valutare la qualità delle dinamiche relazionali che coinvolgono le diverse figure educative diviene dunque un'occasione per soffermarsi e interrogare il consueto, per sostare nella riflessione su aspetti che molto spesso sfuggono nel flusso della complessità quotidiana (Caggio, 2004). Rivedere segmenti significativi della quotidianità scolastica videoregistrati consente di comprenderne maggiormente i significati più profondi, in quanto si crea una situazione apprenditiva di tipo immersivo (Parola, 2012). I filmati, infatti, consentono a ciascun partecipante di vedersi e di ascoltarsi, di osservare il clima complessivo della classe, le reazioni dei bambini alle differenti proposte educative, restituendo la complessità delle situazioni, offrendo diverse piste di lettura e di interpretazione e aprendo nuovi spazi di azione.

In sintesi, "l'artefatto video che viene creato non è una ricetta né una formula: è un'istantanea, un contesto per scambiarsi idee su quel che accade quando la videocamera viene accesa" (Goldman, 2009, p.53). Negli incontri di restituzione, i filmati sono stati quindi utilizzati come spunti di partenza per consentire a ciascun partecipante di rivedersi 
in azione, focalizzando l'attenzione sugli scambi comunicativi e sulle strategie relazionali messe in atto ogni giorno con i bambini, avviando importanti momenti di riflessione, confronto e messa in discussione circa le convinzioni e le ragioni che spesso tacitamente guidano il proprio agire educativo.

In tal senso, Corazza (2012) sostiene che: "la riflessione collettiva sulle immagini girate può essere utile agli attori della situazione educativa per l'attività di metacognizione, per prendere le distanze da se stessi e osservare con spirito scientifico, superando l'autoreferenzialità. Guardarsi in una registrazione video significa accettare un punto di vista diverso, quello dell'occhio della telecamera e della regia. A volte può essere un processo faticoso e sofferto, ma sempre aiuta a riconoscere i propri limiti e a individuare prospettive di miglioramento" (Corazza, 2012, p.19).

In questa prospettiva, gli incontri di restituzione, cuore pulsante dei percorsi di valutazione formativa, arricchiti da una pluralità di linguaggi e di metodologie, divengono dei veri e propri laboratori di sviluppo professionale, nei quali sguardi e saperi differenti si confrontano per co-costruire nuove conoscenze e consapevolezze.

Alimentare il rapporto di ricorsività tra valutazione e riflessione che si viene a realizzare in tali contesti formativi non significa voler uniformare a un modello relazionale prestabilito, ma garantire e valorizzare le unicità e le specificità di ciascun professionista coinvolto, all'interno di una reale condivisione di valori e significati. In tal senso, "affinché i docenti possano qualificarsi come professionisti competenti, devono sviluppare abilità e modi di essere necessari a posizionarsi nella pratica come soggetti impegnati a fare del proprio agire l'oggetto di una indagine rigorosa e di una riflessività continua, ossia sono chiamati a fare ricerca [..] per svincolare la pratica da ogni forma di empiricismo e fare di essa un'azione informata da teorie criticamente costruite ed empiricamente vagliate" (Mortari, 2009, p. 20). 


\section{Narrare e valutare}

Se le modalità appena descritte di valutazione formativa della qualità della relazione educativa fanno riferimento alla videosservazione e all'uso critico di una scala di valutazione, cioè ad uno strumento che scompone analiticamente l'oggetto di osservazione proponendo alcuni descrittori che declinano operativamente una idea di "buona relazione educativa", tecniche differenti possono essere utilizzate - in alternativa o a integrazione di quelle presentate - per esaminare e discutere in equipe il contesto in cui si opera attivando processi di pensiero complessi. Si fa qui riferimento a modi per interrogarsi e rivisitare l'esperienza al fine di mettere in evidenza le logiche che sono sottese all'azione e modalità di documentazione fondate sull'impiego di strategie di scrittura riflessiva. Modalità che valorizzano la narrazione e quindi la ricostruzione soggettiva dell'esperienza - anche nei suoi aspetti emotivi - e l'attenzione nei confronti di episodi che avvengono in situazioni e circostanze specifiche.

La narrazione in questo caso si contrappone a dispositivi di descrizione sistematica e analitica di un fenomeno o una realtà per proporsi, invece, come una forma di approccio all'esperienza che consente di penetrare e comprendere i significati degli eventi vissuti: “Narrare significa costruire contemporaneamente due scenari: quello dell'azione e quello della coscienza dove prendono forma i significati attribuiti all'agire. [...] per saper valorizzare adeguatamente l'attività narrativa occorre avere consapevolezza del fatto che nel racconto di esperienza non c'è un eccesso di soggettivazione, perché il parlare della propria esperienza consente di parlare d'altro; le storie, infatti, sempre riguardano qualcos'altro" (Mortari, 2003, pp. 82-83).

Offrire all'équipe educativa la possibilità di ragionare e confrontarsi sulle narrazioni di eventi particolari proposte dai membri del gruppo significa dunque creare l'opportunità di valutare, nell'accezione più specifica del termine, cioè di assegnare senso e valore, e attivare processi riflessivi sulle azioni e le relazioni che concorrono a definire, a volte in modo almeno in parte inconsapevole, la fisionomia dei contesti educativi. Raccontare, analizzare e problematizzare l'esperienza e la relazione che si ha con gli altri - adulti e bambini - permette di sentirsi componenti di una equipe che comunicano intensamente 
tra di loro, rinegoziando il significato delle pratiche, condividendo e sviluppando un repertorio di stili e di discorsi e una narrazione condivisa su ciò che ha valore nella realtà educativa di cui si è parte. In questo senso i significati del contesto sono ricostruiti di continuo dai comunicanti. E ricostruiti "attraverso processi comunicativi che non sono riducibili all'immediatezza comportamentale, ma che ricorrono anche, insieme, alla mediazione simbolica e alla elaborazione di significati da parte dei singoli comunicanti” (Manghi, 2004, p. 68).

Narrare la relazione, in riferimento agli accadimenti più o meno problematici che si presentano nel quotidiano, apre la possibilità di mettere a fuoco e esplicitare le ragioni che stanno alla base dei comportamenti e di mettere in discussione e negoziare i presupposti pedagogici che fondano determinati atti.

Sotto questo profilo una strategia efficace per sollecitare la riflessione sull'esperienza nell'ambito di una ricognizione individuale o di gruppo su episodi o eventi a cui si è attribuito nell'immediato un particolare significato è quella di sapersi interrogare sull'esperienza stessa attraverso una serie di domande che potremmo definire generative, in quanto promuovono nuovi sguardi sul contesto relazionale che si vive. Infatti "ogni processo di riflessività si attiva mediante la formulazione di domande che il soggetto rivolge a se stesso. Le domande orientano l'attenzione, la ricerca e la selezione d'informazioni e conducono all'esplorazione del mondo intorno a noi" (Sicora, 2010, p. 110). Il ciclo della riflessività di Gibbs (1988) rappresenta un esempio di una sequenza strutturata di domande che può generare riflessività su azioni e situazioni relazionali che per diverse ragioni si ritiene importante analizzare. Possono quindi essere oggetto d'interrogazione e riflessione elementi significativi delle relazioni che un insegnante ha avuto con adulti e bambini, ad esempio il modo con cui un insegnante è intervenuto in una situazione problematica oppure le difficoltà che ha incontrato con un determinato genitore.

Il ciclo della riflessività di Gibbs (cit. in Jasper, 2003) presenta uno schema di domande raggruppate in sei differenti categorie da proporre in successione:

1. Descrizione (che cosa è successo?)

2. Sensazioni (che cosa ho pensato e sentito?) 
3. Valutazione (che cosa c'è stato di positivo e di negativo nell'esperienza?)

4. Analisi (che senso posso dare all'esperienza e alla situazione che si è creata?)

5. Conclusioni (che cos'altro avrei potuto fare?)

6. Piano d'azione (se la situazione si presentasse di nuovo che cosa farei?)

L'analisi dell'evento o episodio su cui s'intende sviluppare una riflessione all'interno dell'èquipe educativa parte dunque dalla richiesta al singolo componente del gruppo di descrivere nel modo più dettagliato possibile quanto è successo. Per poi far emergere anche il suo vissuto emotivo legato all'episodio, che rappresenta un elemento da considerare in quanto può consentire di mettere in luce significati diversi da quelli evidenziati attraverso una indagine razionale. Segue la valutazione in termini di aspetti negativi e positivi dell'esperienza e la lettura del suo significato per mettere a fuoco i motivi di quanto è accaduto. La riflessione si completa ragionando sulle possibili ricadute operative sia in riferimento a quanto si poteva fare di diverso in quello specifico contesto, sia a ipotesi di azioni differenti nel caso si ripresentassero situazioni simili.

Il modello proposto da Gibbs può essere applicato in vario modo, infatti "la riflessione può essere condotta tra sé e sé, cioè su un piano sostanzialmente mentale e meditativo, oppure verbalmente, cioè parlando con altre persone in merito a un evento ritenuto significativo. C'è poi un ulteriore ambito di sviluppo della riflessività ed è quello rappresentato dalla scrittura" (Sicora, 2010, p. 114).

Da questo punto di vista uno strumento di scrittura riflessiva estremamente utile e assolutamente sostenibile sotto il profilo dei tempi e dei carichi di lavoro che comporta è il diario di bordo. Uno strumento che permette di documentare e costruire memoria dell'esperienza quotidiana per poterla fare oggetto di una riflessione a posteriori anche nell'ambito del gruppo di lavoro. Si tratta quindi di lasciare traccia sistematicamente di quanto accade giornalmente, senza evitare di esplicitare il modo con cui si sono interpretati gli eventi e la risonanza che gli stessi hanno avuto sul piano emozionale, per analizzarlo successivamente e incrementare i livelli di consapevolezza sul proprio agito.

Il diario, che richiede dei tempi regolari di scrittura, può contenere "varie tipologie di dati" (Mortari, 2003, p. 94): descrizioni dettagliate di situazioni realizzate anche attraverso l'uso dell'osservazione, narrazioni di avvenimenti e episodi che sono successi e 
delle circostanze in cui sono accaduti, valutazioni che riguardano il proprio modo di comportarsi in momenti particolari, intuizioni che si possono avere in determinate occasioni e che è utile annotare, ricostruzioni dei propri vissuti emotivi e ipotesi su possibili interventi o strategie relazionali da mettere in campo nel futuro. L'analisi di quanto scritto nel diario può sollecitare riflessioni che riguardano sia le implicazioni teoriche delle proprie pratiche, sia elementi di dubbio o questioni che si vogliono approfondire o eventuali azioni che possono contribuire a migliorare la situazione sotto esame.

Il diario costituisce, comunque, uno strumento essenziale per creare l'opportunità di un confronto dialogico costruttivo sulle relazioni e sulle pratiche all'interno dell'équipe educativa. Si può dire, infatti, che "non si apprende il pensiero critico al di fuori di uno spazio pubblico [... ]. Quando gli altri ascoltano i nostri resoconti e possono riflettere su di essi è possibile trovarsi a confronto con altre interpretazioni differenti dalla nostra, che ci spingono a costruire una nuova versione interpretativa del nostro agire, la quale rappresenta il presupposto da cui può scaturire la messa a punto di modelli alternativi di pratica" (Mortari, 2003, pp. 110-111).

Il diario di bordo, così come altri strumenti di scrittura riflessiva, rappresenta dunque un modo efficace per indagare e analizzare criticamente la relazione educativa sollecitando una reale pratica riflessiva all'interno del gruppo di lavoro. In questo senso i due approcci proposti, che valorizzano attraverso strumenti differenti i processi di osservazione e narrazione, costituiscono forme diverse e complementari per esplorare e far emergere i significati delle componenti relazionali del contesto, attivando dinamiche di confronto tra i membri dell'èquipe che possono portare ad una comprensione più profonda delle implicazioni formative dei propri atti comunicativi e, di conseguenza, alla costruzione di uno stile educativo maggiormente condiviso. 


\section{Riferimenti}

Balduzzi Lucia, Manini Milena (a cura di) (2013), Professionalità e servizi per l'infanzia, Roma, Carrocci editore.

Becchi Egle (1978). "Il sapere che entra nella scuola”. Riforma della scuola, 6/7, 9-11.

Bove Chiara (2009), Ricerca educativa e formazione: contaminazioni metodologiche, Milano, Franco Angeli.

Bowlby John (1969), Attachment and loss: vol. 1. Attachment, Basic Books, New York, trad. it. Boringheri, Torino, 1989.

Cabell Sonia et al. (2013), "Variation in the effectiveness of instructional interactions across preschool classroom settings and learning activities", in Early Childhood Research Quarterly, 28, 4, pp. 820-830.

Cadima Joana, Leal Teresa, Burchinal Margaret (2010), "The quality of teacher-student interactions: associations with first graders' academic and behavioral outcomes", in Journal of School Psychology, 48, pp. 457-482.

Caggio Francesco, Mantovani Susanna (a cura di) (2004), Famiglie, bambini, educatrici: esplorazioni del consueto, Azzano San Paolo (Bg), Edizioni Junior.

Cappa Francesco (2014). "Introduzione”, in Cappa Francesco (a cura di), Metodo e qualità dell'esperienza scolastica, Milano, Franco Angeli.

Cescato Silvia, Bove Chiara, Braga Piera (2015), “Video, formazione e consapevolezza. Intrecci metodologici”, in Form@re, 2, 15, pp. 61-74.

Colicchi Enza (2005). "Note su intenzionalità educativa e razionalità", in Cambi Franco (a cura di), Le intenzioni nel processo formativo, Tirrenia (Pisa), Edizioni del Cerro.

Connell James, Wellborn James (1991), “Competence, autonomy and relatedness: A motivational analysis of self-system processes", in Megan Gunnar, Alan L. Sroufe (a cura di), Minnesota symposium on child psychology, NJ Erlbaum, Hillsdale, v. 23, pp. 43-77.

Corazza Laura (2012), “Il video, un mediatore per l'apprendimento”, in Form@re, 79, 12, pp. 13-21.

Curby Timothy et al. (2009), "The relations of observed pre-k classroom quality profiles to children'achievement and social competence”, in Early Education and Development, 20, pp. 346-372. 
Dower Jason, Sabol Terri, Hamre Bridge (2010), "Teacher-Child interactions in the classroom: toward a theory of whitin and cross-domain links to children's developmental outcomes", in Early Education and Development, 21, 5, pp. 699-723.

Downer Jason et al. (2012), "A course on effective teacher-child interactions: effects on teacher beliefs, knowledge, and observed practice", in American Educational Research Journal, 49, 1, pp. 88-123.

Fabbri Loretta (2007). Comunità di pratiche e apprendimento riflessivo. Roma, Carocci. Franceschini Giuliano, Borin Paolo, Il curricolo nella scuola dell'infanzia, Roma, Carocci.

Gibbs Graham (1988), Learning by doing: a guide to teaching and learning methods, Oxford, Further Education Unit, Oxford Polytechnic, cit. in Jasper Melanie (2003), Beginning reflective practice (Foundation in nursing and health care), Cheltenham, Nelson Thornes Ltd.

Goldman Ricki, Pea Roy, Barron Brigid, Derry Sharon J. (a cura di) (2007), Videoricerca nei contesti di apprendimento. Teorie e metodi, trad. it. Raffaello Cortina, Milano, 2009. Hamre Bridge et al. (2013), "Teaching through interactions. Testing a Developmental Framework of Teacher Effectiveness in over 4.000 classrooms", in The Elementary School Journal, 113, 4, pp. 462-487.

Hanks William (1991), “Prefazione”, in Lave Jean, Wenger Etienne, L'apprendimento situato, trad. it. Erickson, Trento, 2006.

Hinde Robert (1981), “Commentary”, in Human Development, 33, pp. 283-288.

LoCasale-Crouch Jennifer et al. (2007), "Observed classroom quality profiles in statefunded pre-kindergarten programs and associations with teacher, program, and classroom characteristics", in Early Childhood Research Quarterly, 22, 1, pp. 3-17.

Manghi Sergio (2004), La conoscenza ecologica, Milano, Raffaello Cortina Editore.

Mantovani Susanna (a cura di) (1989), La scuola del bambino: commento ai nuovi orientamenti per la scuola materna della Commissione Scurati, Bergamo, Juvenilia.

Mortari Luigina (2003), Apprendere dall'esperienza, Roma, Carocci.

Mortari Luigina (2009), Ricercare e riflettere. La formazione del docente professionista, Roma, Carocci.

Neri Sergio, (2001), Guardare vicino e lontano. Qualità e prospettive della scuola dell'infanzia, Milano, Fabbri editori. 
Pakarinen Eija et al. (2010), "A Validation of the Classroom Assessment Scoring System in Finnish Kindergartens”, in Early Education and Development, 21, 1, pp. 95-124.

Pakarinen Eija et al. (2012), "A Validation of the Early Childhood Classroom Observation Measure in Finnish and Estonian Kindergartens", in Early Education and Development, 23, pp. 323-350.

Palmieri Cristina (2014). "Metodo e qualità dell'esperienza scolastica: percorsi di ricerca e formazione”, in Cappa Francesco (a cura di), Metodo e qualità dell'esperienza scolastica, Milano, Franco Angeli.

Parola Alberto, (2012), Regia educativa. L'insegnante osservatore tra percorsi "in ricerca": linguaggi e strategie, Roma, ARACNE editore.

Pattison Cassandra et al. (2014), "Emotional Climate and Behavioral Management during Sleep time in Early Childhood Education settings", in Early Childhood Research Quarterly, 29, pp. 660-668.

Pianta Robert (1999), La relazione bambino-insegnante: aspetti evolutivi e clinici, trad. it. Raffaello Cortina, Milano, 2001.

Pianta Robert et al. (2005), "Features of Pre-Kindergarten Programs, Classrooms, and Teachers: Do they predict observed classroom quality and child-teacher interactions?", in Applied Developmental Science, 9, 3, pp. 144-159.

Pianta Robert, La Paro Karen, Hamre Bridge (2008), Classroom Assessment Scoring System (CLASS) manual, Brookes, Baltimore.

Pianta Robert, Hamre Bridge (2009), "Conceptualization, Measurement and Improvement of Classroom Processes: standardized observation can leverage capacity", in Educational Researcher, 38, 2, pp. 109-119.

Salminen Jenni et al. (2012), “Observed Classroom Quality Profiles of Kindergarten Classrooms in Finland", in Early Education and Development, 23, pp. 654-677.

Savio Donatella (2011), "Il gioco e la partecipazione del bambino: una sfida educativa e i suoi nodi”, in Anna Bondioli, Donatella Savio (a cura di), Partecipazione e Qualità. Percorsi di condivisione riflessiva nei servizi per l'infanzia di Modena, Parma, Edizioni Junior-Spaggiari Edizioni, pp. 205-251.

Sicora Alessandro (2010), Errore e apprendimento nelle professioni di aiuto, Santarcangelo di Romagna (RN), Maggioli. 
Terzi Nice (2017), “Cura e relazione educativa: promuovere l'apprendimento nella prospettiva dello o/6", in Claudia Lichene (a cura di), Conoscere lo 0-6, I Quaderni, 3, Bergamo, Zeroseiup edizioni, pp. 53-57.

Tochon Francois Victor (2009). "Dai video-casi alla video-pedagogia. Una cornice teorica per il video-feed-back e la riflessione con i video nella pratica della ricerca pedagogica”. In Ricki Goldman, Roy Pea, Brigid Barron, Sharon Derry, Videoricerca nei contesti di apprendimento.Teorie e metodi (pp. 83-101), Milano, Raffaello Cortina.

Vitiello Virginia, Hadden Sarah (2014), CLASS System. Implementation guide. Aligned improvement solutions, Charlottesville, VA, Teachstone.

Von Suchodoletz Antje et al. (2014), "A typical morning in preschool: Observations of teacher-child interactions in German preschools", in Early Childhood Research Quarterly, 29, pp. 509-519. 